\title{
Brn-3a and Brn-3b are differentially expressed in recurrent respiratory papillomatosis associated to HPV-6 and HPV-11 infection.
}

\author{
Sanchez-Gonzalez Maria Teresa ${ }^{1}$, Marino-Martinez Ivan Alberto ${ }^{*}$, Treviño-Gonzalez Jose Luis ${ }^{2}$, \\ Ponce-Camacho Marco Antonio ${ }^{3}$, Santos-Lartigue Ramiro ${ }^{4}$, Martinez-Vite Raul ${ }^{5}$, Villagomez-Ortiz \\ Vicente Jose $^{6}$, Ortiz-Lopez Rocio ${ }^{7}$, Rojas-Martinez Augusto ${ }^{7}$
}

${ }^{1}$ Autonomous University of Nuevo León, Center for Research and Development in Health Sciences (CIDICS), Monterrey, Nuevo León, México

${ }^{2}$ Autonomous University of Nuevo León, Otorhinolaryngology Service, School of Medicine, Monterrey, Nuevo Leon, México

${ }^{3}$ Autonomous University of Nuevo León, Pathology and Cytology Service, School of Medicine, Monterrey, Nuevo León, México

${ }^{4}$ Otorhinolaryngology Service, Hospital Materno Infantil, Secretaría de Salubridad y Asistencia, Monterrey Nuevo León, México

${ }^{5}$ Department of Otorhinolaryngology, Hospital Los Ángeles Valle Oriente, San Pedro Garza García, Nuevo León, México

${ }^{6}$ Department of Otorhinolaryngology, Hospital Zambrano Hellion, San Pedro Garza García, Nuevo León, México

${ }^{7}$ Instituto Tecnologico de Estudios Superiores de Monterrey, Escuela de Medicina y Ciencias de la Salud, Monterrey, Nuevo León, México

\begin{abstract}
Brn-3a and Brn-3b are transcription factors expressed mainly in somatosensory neurons during embryonic stage; but they are absent in adulthood. Both share the same DNA binding site with an antagonist effect. Brn-3a expression has been reported 300 fold increased in Cervical Intraepithelial Neoplasia grade 3 (CIN3) compared with normal cervix tissue; some studies suggest that Brn-3a may increase the expression of HPV onco-proteins E6 and E7 by interaction with the promoter region of some high risk HPV genotypes. In this study, we analyzed the expression of Brn-3a and Brn-3b in laryngeal tissue biopsies from patients with Recurrent Respiratory Papillomatosis (RRP) and in volunteers' health tissue. HPV was detected in all RRP samples and only HPV-6 and HPV-11 were detected in the samples of RRP. Brn-3a was expressed in $87 \%$ of the samples and Brn-3b in $45 \%$ coexpressed with Brn-3a. Brn-3a is expressed in laryngeal papillomas of patients with RRP diagnostic; but not in normal laryngeal tissue. The most of the samples expresses Brn-3a and only half weakly expresses Brn-3b; which suggests that the antagonist role of Brn-3b over Brn-3a is absent in this process.
\end{abstract}

Keywords: Brn-3a, Brn-3b, HPV, Recurrent respiratory papillomatosis.

Accepted on June 12, 2018

\section{Introduction}

Brn-3a/POU4f1, Brn-3b/POU4f2 and Brn-3c/POU4f3 are members of the POU transcription factors family which play an important role in mammalian early embryonic development. They are expressed principally in subsets of overlapping visual, auditory and somatosensory neurons [1-3]. Although Brn-3a and Brn-3b share same DNA binding motives, it has been reported that these two factors exert opposite effects on their targets transcription [4-6]. Brn-3 transcription factors interact additively with during Retinal Ganglion Cell (RGC) generation, but have distinct effects on the specification of the morphological features necessary to establish RGC cell types. Brn-3b affects Brn-3a and Brn-3c positive RGCs, Brn-3b loss affects survival, and axon and dendritic arbor features of Brn-3a positive RGCs, including those that normally do not express Brn3b in the adult [7].

Besides its participation in neuronal embryonic development, some reports suggest that Brn-3a and Brn-3b can be involved 
in cancer development through transcriptional regulation of some pro-apoptotic and suppressor tumor genes such as Bcl-2, $B c l-X$ and $B R C A-1$ [8-10]. Expression of Brn-3a and Brn-3b in adult tissues is rare; however the expression of Brn-3a and Brn- $3 \mathrm{~b}$ in neuroendocrine tumors, melanoma, prostate, ovarian and breast cancer have been reported. [10-14]. Latchman et al. described functionally antagonistic effects, with Brn-3a activating specific gene promoters which are repressed by Brn- $3 \mathrm{~b}$ during the process of neuroblastoma differentiation and proliferation $[15,16]$. It is the first report of the possible participation of ratio Brn-3a/Brn-3b in cancer development. Latchman et al. demonstrated the Brn-3a overexpression in CIN3. [17] They also reported that Brn-3a and Brn-3b bind to ATGCAATT, a sequence which is present in the URR of some HPV-16 and HPV-18 with antagonistic effects, with Brn-3a activating and Brn-3b repressing HPV expression in cervix cell lines [18].

Recurrent Respiratory Papillomatosis (RRP) is a histological benign and chronic larynx, trachea, and bronchi disease. It is the most frequent benign neoplasm in childhood and is associated to HPV-6 and HPV-11 infection. Although HPV-6 and HPV-11 are not considered high risk HPVs, RRP may evolve and compromise the larynx function, and eventually, it may evolve to squamous cell carcinoma [19-22].

There are not expression reports of Brn-3a or Brn-3b in normal or unhealthy oropharyngeal tissue. It is clear that Brn-3a may induce HPV-16 and 18 expressions, increasing their oncogenic potential. Changes in the URR, HPV-6 and 11 sequences, may allow regulation of their viral promoter by Brn-3a, facilitating the progressive increase in the grade of disorder in the papillomas or to spontaneous squamous cell carcinoma development.

This study's aim was to analyze the Brn-3a and Brn-3b expression in fresh laryngeal tissue from RRP patients. Additionally it was performed detection of HPV presence and specific genotypes such HPV-6, HPV-11, HPV-16 and HPV-18.

\section{Materials and Methods}

All procedures and tests performed during this work were approved by the Institutional Review Board (IRB) of the Hospital Universitario UANL with number OT12-003.

RRP biopsies and laryngeal brush samples: Fresh biopsies were collected in the Service of Otorhinolaryngology at the Hospital Universitario UANL (Nuevo Leon, Mexico). Laryngeal papillomas $(n=11)$ were collected by direct microlaryngoscopy surgery with sub-epithelial resection. Each specimen was divided into two pieces of $2 \mathrm{~mm}$ used for DNA and RNA isolation respectively. Both specimens were stored at $-70^{\circ} \mathrm{C}$ until pathologist observation and nucleic acid extraction. The pathologist confirmed papillomas through histological observation. Normal laryngeal tissue expression was evaluated in brush samples from asymptomatic volunteers, negatives for $\operatorname{HPV}(\mathrm{n}=10)$.
DNA and RNA isolation: Nucleic acids were isolated from fresh RRP biopsies and normal laryngeal brush samples. Nucleospin Tissue Genomic DNA Purification Kit and Nucleospin RNA of Macherey-Nagel (Duren, Germany) were used to perform the genomic DNA and total RNA isolation respectively, following protocols of the manufacturer. Genomic DNA and total RNA were quantified in the spectrophotometer Nanodrop 2000 (Thermo Scientific) and diluted to final concentration of $60 \mathrm{ng} / \mu \mathrm{L}$ for DNA, and $30 \mathrm{ng} / \mu \mathrm{L}$ for RNA. Once diluted, nucleic acids were stored at $-80^{\circ} \mathrm{C}$ until required.

PCR for HPV detection and genotyping: The presence of HPV in RRP samples and oropharyngeal brush was detected by end-point PCR using universal MY (MY06/MY09) oligonucleotides. PCR reactions were performed using homedesigned HPV type specific oligonucleotides to detect a LCR region; 5'-TAT TCG TAC CGG TGT TAA GCGCC-3'/5'TTG GCA GGG CAA TGT GTA CCTG-3' for HPV-6, 5'GCT GTG TCT AAG CCC TCT ACAG-3'/5'- GTA CCT TGG CAC ACA ACA TATGG-3' for HPV-11, 5'-ACC TCA TCT ACC TCT ACA ACTGC-3'/5'- GGC AAG CAG TGC AGG TCAGG-3' for HPV-16 and 5'-AAC CTG CCA AGC GTG TGC GTG-3'/5'-TAA GCC AAA GGC AAC CGA AATCG -3' for HPV-18. Oligonucleotides for Brn-3a, Brn-3b, E2-HPV6, E2-HPV11, E6-HPV6 and E6-HPV11 analyses were 5'-TGG CGT CCA TCT GCG ACTC-3'/5'-CTCA GGC TTG TTC ATT TTCTC-3' for Brn-3a, 5'-GGA GAA GAA GCG CAAGC-3'/5'-TCT GGA GAG GCC AAG AGTC-3' for Brn-3b, 5'-AAG CGT TTA GAT GCG TGC CAG-3'/5'ATT GTA TTG TTT GCA CAG CCATC-3' for E2-HPV6, 5'ATA CAC AAA CAC ATT ATG CAT TGG-3'/5'-ATA TGT GTC CAT ACC ACA TACTC-3' for E2-HPV11, 5'-ATG CCT CCA CGT CTG CAACG-3'/5'-ATG TTG TCC AGC AGT GTA GGC-3' for E6-HPV6 and 5'-TAA AGA TGC CTC CAC GTC TGC-3'/5'-AAG TCT TCC ATG CAT GTT GTCC-3' for E6-HPV11.

PCRs were performed using Green Go-Taq 2X MasterMix of Promega (Madison, WI, USA) and all oligonucleotides were ordered to IDT (Coralville, Iowa, USA). Annealing temperatures for MYs and all home-designed oligonucleotides were $60^{\circ} \mathrm{C}$ and $68^{\circ} \mathrm{C}$ respectively. Genomic DNA from HeLa (HPV-18), SiHa (HPV-16) and clinical samples previously identified as positives to the presence of HPV-6 or HPV-11 were used as positive controls for PCR.

cDNA synthesis: cDNA synthesis for expression analysis of Brn-3a and Brn-3b was made using oligo-dT method. Retrotranscription reactions were performed with Superscript III Reverse Tanscriptase of Invitrogen (Waltham, MA, USA) and $60 \mathrm{ng}$ of total RNA as template. Manufacturer's conditions were followed.

\section{Results}

HPV-11 is the most prevalent in our RRP population. HPV presence was confirmed by end-point PCR using degenerated MY oligonucleotides in the total of the RRP samples (11/11) (Figure 1a) and absent in normal laryngeal brush samples 
(0/10). Only genotypes HPV-6 and HPV-11 were identified in RRP samples. HPV-11 was the most prevalent with $73 \%(8 / 11)$ (Figure 1b) and HPV-6 was present in 27\% (3/11) (Figure 1c) of the RRP samples. HPV-16 and HPV-18 were not detected using specific end-point PCR (Figures 1d and 1e). There was no co-infection with HPV-6 and HPV-11 in any sample.

Brn-3a and Brn-3b are expressed in RRP tissue. Even when Brn-3a and Brn-3b are neuronal, embryonic specific transcription factors, expression Brn-3a and Brn-3b was detected in biopsies from RRP patients. The $82 \%(9 / 11)$ of RRP samples were positive for Brn-3a expression and $45 \%$ (5/11) co-expressed Brn-3a and Brn-3b. The 67\% (6/9) of Brn-3a positive samples were associated to HPV-11 and only $33 \%(3 / 9)$ were associated to HPV-6. 3 Brn-3a (+)/HPV-11 (+) and one Brn-3a $(+) / H P V-6(+)$ did not express Brn-3b. The $60 \%(3 / 5)$ of Brn-3b positive samples were associated to HPV-11 and 40\% (2/5) were associated to HPV-6. All Brn-3b (+) samples co-expressed Brn-3a (Figure 2). Only the $18 \%$ $(2 / 11)$ of RRP samples showed no expression of Brn-3a and Brn-3b.

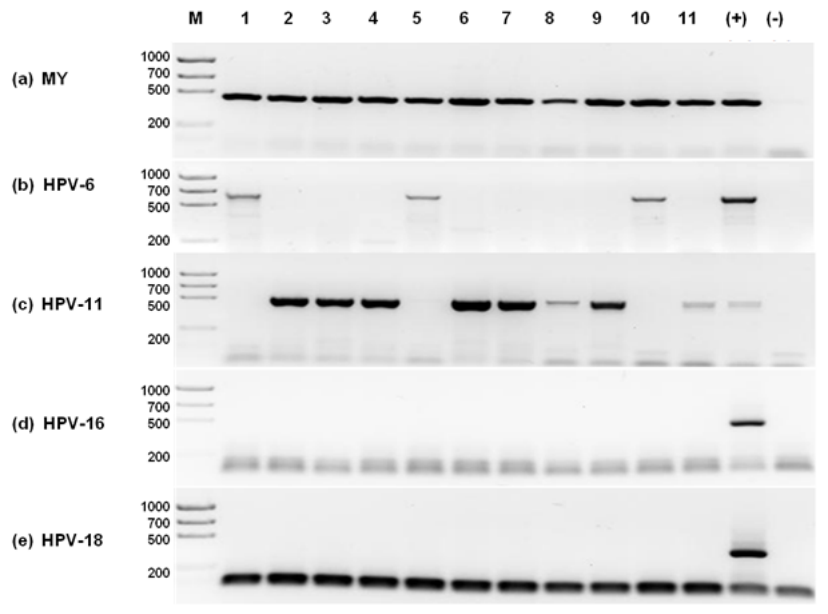

Figure 1. HPV detection and genotyping for HPV types 6, 11, 16 and 18. (a) End-point PCR for HPV detection using primers MY06/MY09 in larynx papillomas (1-11). End-point PCR for genotyping; (b) HPV-6; (c) HPV-11; (d) HPV-16; (e) HPV-18 in larynx papillomas (1-11). Each PCR includes positive (+) and negative control (-).

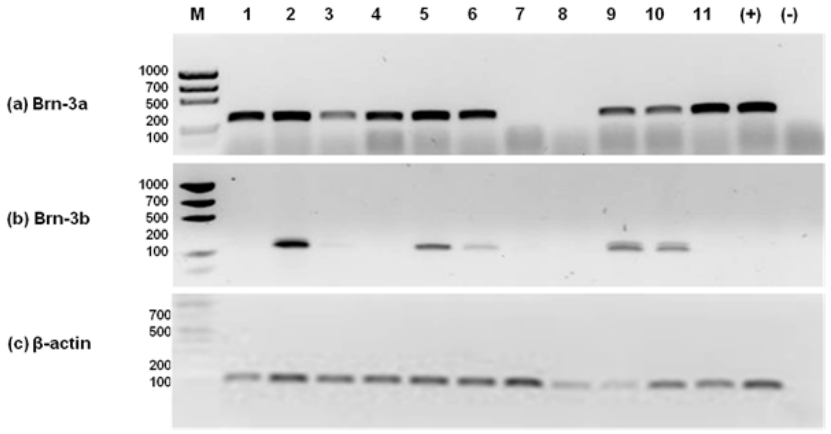

Figure 2. Expression of Brn-3a and Brn-3b in laryngeal papillomas. End-point PCR amplification of the messengers: (a) Brn-3a; (b) Brn-3b; expressed in laryngeal papillomas samples (1-11); (c) $\beta$-actin was used as endogenous gene.

\section{Discussion}

Brn-3a and Brn-3b are neuronal embryonic transcription factors that exhibit opposite effects, related to early embryonic development, and are absent in adulthood. Morris et al. reported that, Brn-3a binds HPV-16 promoter (URR) and activates transcription [6]. Ndisang et al. identified that Brn-3a is expressed in normal cervix tissue, and is overexpressed 300fold in cervical intraepithelial neoplasia grade 3 (CIN3) lesions compared to normal tissue [17]. Both observations suggest that, Brn-3a is an important cellular factor that elevates risk of cervix transformation if it is infected with HPV variants that are activated by Brn-3a, by increasing levels of transforming oncogenes E6 and E7. However, HPV-16 is more prevalent in cervix infection but traditionally not prevalent in larynx, in which we demonstrated Brn-3a expression compared to normal tissue.

Ndisang et al. suggests that, basal expression in normal cervix tissue is not related to the oncogenic process, and that it is necessary the presence of HPV for carcinoma development $[17,18]$. However, recently Brn-3a has been described in other HPV not related types of cancers, such as prostate cancer and melanoma [12,15]. Participation of Brn-3a in apoptosis regulation is well recognized since this neuronal transcription factor blocks promoters of two pro-apoptotic p53 targets such as Noxa and Bax [22]. By contrary, two anti-apoptotic genes such as $B c l-x$ and $B c l-2$ are repressed by $\mathrm{p} 53$ and stimulated by Brn-3a $[8,9]$. The role of Brn-3a in cancer development may not be dependent of HPV, but in some tissues where HPV infection is the onset for cell transformation, the interaction between Brn-3a and some HPV variants increase the risk for cancer establishment.

In this study we demonstrate that Brn-3a is expressed in laryngeal papillomas of RRP diagnosed patients; but not expressed in normal laryngeal tissue. The most of the patients expresses Brn-3a (9/11) and only half (5/11) weakly expresses Brn-3b. This last known Brn-3a antagonist competes for the same binding sites on their targets promoters. Besides, these results need to be corroborated in a bigger population with a quantifiable technique; these results suggest that HPV infected tissues express Brn-3a rather than Brn-3b. There are no previous reports of Brn-3a and Brn-3b expressions in health or pathologic laryngeal tissue. Morris et al. found that, transfecting equal amounts of a vector expressing Brn-3b and a vector expressing Brn-3a; the expression of a reporter due to Brn-3a binding to URR of HPV16 was inhibited. This inhibition was reverse to the ratio Brn-3a/Brn-3b [6]. Our observations by end-point PCR show that, apparently there is more messenger of Brn-3a than Brn-3b, which may suggest that in laryngeal papillomas, Brn-3b is not able to inhibit Brn-3a. The observed effect of Brn-3b and Brn-3a on URR of HPV16 by Morris et al. [6] is important since the virus expression may be increased by Brn-3a in absence of Brn-3b. Our results suggest that Brn-3a may control gene expression in RRP tissue without Brn-3b repression. This effect of Brn-3a may be on viral genes of some HPV variants, and not only on cellular genes that present the binding motif for Brn-3a and 
Brn-3b on their promoters. However there is no evidence that the URR in HPV6 and 11 present the motif for Brn-3a and Brn-3b. If it is true that URR of HPV6 and HPV11 respond to Brn-3a, our findings may correlate with the observations of Latchman et al. [16] with the difference that we are observing the same behaviour of Brn-3a on a tissue lesion related to low risk HPVs.

Most of the reports that describe the relation between HPV infection and Brn-3a expression are in cervix. In our study, we were not able to detect HPV16 and HPV18 (the most prevalent HPV genotypes in cervix) in laryngeal papillomas, these HPV genotypes have URR variants that respond to Brn-3a and Brn-3b presence [6]. Indeed, the most of patients showed infection by HPV11 (8/11) and only few patients showed HPV6 (3/11) infection. It will be interesting to investigate the interaction of Brn-3a with promoter regions of HPV considered as low risk virus such as HPV-6 and HPV-11, understanding that a low risk HPV that responds to Brn-3a may act as high risk HPV. The prevalence of common laryngeal HPV genotypes such as HPV-6 and HPV-11 generates the next question: Does low risk HPVs take advantage of changes in Brn-3a/Brn-3b ratio?

Since up-regulation of Brn-3a happens in two different but at the same time related lesions such as CIN3 and larynx papilloma, which have in common presence of HPV, we may think that increase in Brn-3a levels and decrease of Brn-3b levels are directly caused by HPV infection. However, two samples that do not express Brn-3a and Brn-3b suggest that presence of HPV is not sufficient to trigger Brn-3a and Brn-3b expression. Interestingly, from the six HPV11 positive papillomas that express Brn-3a, only three co-expresses Brn-3b, while in three HPV6 positive papillomas expressing Brn-3a, two co-expresses Brn-3b. It may suggest that some HPV genotypes are able to suppress the Brn-3b expression.

It is interesting that, embryonic neuronal specific transcription factor Brn-3a is up-regulated in a premalignant lesion such as $\mathrm{CIN} 3$, and in a benign tumor of non-neuronal tissue such as laryngeal papillomatosis, even more interesting would it be to know the roll of Brn-3a/Brn-3b ratio in RRP clinical outcome.

\section{Acknowledgment}

Thanks to LIC. Sandra Margarita Chapa-Hernandez and ING. Andrés Alberto Garcia-Moreno from RSC (Reteach and Study Center) School, for revising English version of manuscript.

\section{References}

1. Xiang M, Zhou L, Macke JP, Yoshioka T, Hendry SH, Eddy RL, Shows TB, Nathans J. The Brn-3 family of POU-domain factors: primary structure, binding specificity, and expression in subsets of retinal ganglion cells and somatosensory neurons. J Neurosci 1995; 15: 4762-4785.

2. Xiang M, Gan L, Zhou L, Klein WH, Nathans J. Targeted deletion of the mouse POU domain gene Brn-3a causes selective loss of neurons in the brainstem and trigeminal ganglion, uncoordinated limb movement, and impaired suckling. Proc Natl Acad Sci USA 1996; 15: 11950-11955.

3. Xiang M, Gan L, Li D, Chen ZY, Zhou L, O'Malley BW Jr, Klein W, Nathans J. Essential role of POU-domain factor Brn-3c in auditory and vestibular hair cell development. Proc Natl Acad Sci USA 1997; 94: 9445-9450.

4. Budhram-Mahadeo V, Parker M, Latchman DS. POU transcription factors Brn-3a and Brn-3b interact with the estrogen receptor and differentially regulate transcriptional activity via an estrogen response element. Mol Cell Biol 1998; 18: 1029-1041.

5. Theil T, Rödel B, Spiegelhalter F, Möröy T. Short isoform of POU factor Brn-3b can form a heterodimer with Brn-3a that is inactive for octamer motif binding. $\mathrm{J}$ Biol Chem 1995; 270: 30958-30964.

6. Morris PJ, Theil T, Ring CJ, Lillycrop KA, Moroy T, Latchman DS. The opposite and antagonistic effects of the closely related POU family transcription factors Brn-3a and Brn-3b on the activity of a target promoter are dependent on differences in the POU domain. Mol Cell Biol 1994; 14: 6907-6914.

7. Shi M, Kumar SR, Motajo O, Kretschmer F, Mu X, Badea TC. Genetic interactions between Brn3 transcription factors in retinal ganglion cell type specification. PLoS One 2013; 8: e76347.

8. Budhram-Mahadeo V, Morris PJ, Smith MD, Midgley CA, Boxer LM, Latchman DS. p53 suppresses the activation of the Bcl-2 promoter by the Brn-3a POU family transcription factor. J Biol Chem 1999; 274: 15237-15244.

9. Sugars KL, Budhram-Mahadeo V, Packham G, Latchman DS. A minimal Bcl-x promoter is activated by Brn-3a and repressed by p53. Nucleic Acids Res 2001; 29: 4530-4540.

10. Budhram-Mahadeo V, Ndisang D, Ward T, Weber BL, Latchman DS. The Brn-3b POU family transcription factor represses expression of the BRCA-1 anti-oncogene in breast cancer cells. Oncogene 1999; 18: 6684-6691.

11. Leblond-Francillard M, Picon A, Bertagna X, de Keyzer Y. High expression of the POU factor Brn3a in aggressive neuroendocrine tumors. J Clin Endocrinol Metab 1997; 82: 89-94.

12. Diss JK, Faulkes DJ, Walker MM, Patel A, Foster CS, Budhram-Mahadeo V, Djamgoz MB, Latchman DS. Brn-3a neuronal transcription factor functional expression in human prostate cancer. Prostate Cancer Prostatic Dis 2006; 9: 83-91.

13. Ahmed N, Latifi A, Riley CB, Findlay JK, Quinn MA. Neuronal transcription factor Brn-3a (1) is over expressed in high-grade ovarian carcinomas and tumor cells from ascites of patients with advanced-stage ovarian cancer. $\mathbf{J}$ Ovarian Res 2010; 3: 1-12.

14. Fujita R, Ounzain S, Wang AC, Heads RJ, BudhramMahadeo VS. Hsp-27 induction requires POU4F2/Brn-3b 


\section{$H P V-11$ infection}

$\mathrm{TF}$ in doxorubicin-treated breast cancer cells, whereas phosphorylation alters its cellular localisation following drug treatment. Cell Stress Chaperones 2011; 16: 427-439.

15. Hohenauer T, Berking C, Schmidt A, Haferkamp S, Senft D, Kammerbauer C, Fraschka S, Graf SA, Irmler M, Beckers J, Flaig M, Aigner A, Höbel S, Hoffmann F, Hermeking H, Rothenfusser S, Endres S, Ruzicka T, Besch R. The neural crest transcription factor Brn3a is expressed in melanoma and required for cell cycle progression and survival. EMBO Mol Med 2013; 5: 919-934.

16. Smith MD, Latchman DS. The functionally antagonistic POU family transcription factors Brn-3a and Brn-3b show opposite changes in expression during the growth arrest and differentiation of human neuroblastoma cells. Int J Cancer 1996; 67: 653-660.

17. Ndisdang D, Morris PJ, Chapman C, Ho L, Singer A, Latchman DS. The HPV-activating cellular transcription factor Brn-3a is overexpressed in CIN3 cervical lesions. J Clin Invest 1998; 101: 1687-1692.

18. Ndisang D, Faulkes DJ, Gascoyne D, Lee SA, Ripley BJ, Sindos M, Singer A, Budhram-Mahadeo V, Cason J, Latchman DS. Differential regulation of different human papilloma virus variants by the POU family transcription factor Brn-3a. Oncogene 2006; 25: 51-60.
19. Intakorn $\mathrm{P}$, Sonsuwan N. Human papillomatosis genotyping and severity in patients with recurrent respiratory papillomatosis. J Med Assoc Thai 2014; 97: 136-141.

20. Carifi M, Napolitano D, Morandi M, Dall'Olio D. Recurrent respiratory papillomatosis: current and future perspectives. Ther Clin Risk Manag 2015; 11: 731-738.

21. Gupta S, Gupta S. Role of human papillomavirus in oral squamous cell carcinoma and oral otentially malignant disorders: A review of the literature. Indian J Dent 2015; 6: 91-98.

22. Hudson CD, Morris PJ, Latchman DS, BudhramMahadeo VS. Brn-3a transcription factor blocks p53mediated activation of proapoptotic target genes Noxa and Bax in vitro and in vivo to determine cell fate. $\mathrm{J}$ Biol Chem 2005; 280: 11851-11858.

\section{*Correspondence to}

Marino-Martinez Ivan Alberto

Autonomous University of Nuevo León

Center for Research and Development in Health Sciences (CIDICS),

México 